\title{
Emphydematous pyelonephritis - Treat the patient, not the scan
}

\author{
Jun Yang Chia, Ho Yee Tiong, Joe Lee King Chien \\ Department of Urology, National University Health System, Singapore
}

Received: July 14, 2015

Accepted: November 1, $2015 \quad$ Online Published: November 3, 2015

DOI: $10.5430 /$ css.v2n1p49

URL: http://dx.doi.org/10.5430/css.v2n1p49

\begin{abstract}
Background: Emphysematous pyelonephritis (EPN) is a severe, necrotizing infection of the renal parenchyma. Until the late 1960s, these patients were treated with nephrectomies as EPN is associated with a high rate of mortality. It is a diagnosis based on computed tomography (CT) scan of the patient and based on the results, can be classified according to the severity of the disease. In our case report, we present a patient with seemingly extensive EPN on CT scan who was treated successfully with intravenous antibiotics (IV) and percutaneous drainage.

Methods: Together with current literature, our case study adds to increasing evidence which supports how carefully selected patients can be treated with the above modalities.

Results: We present a 75-year-old diabetic Indian gentleman who came to us with right iliac fossa pain and was noted to have a positive right renal punch. CT scan revealed extensive gas in the right kidney. Considering that he was clinically stable amongst other factors, he was started on appropriate IV antibiotics and underwent a percutaneous nephrostomy tube insertion. Subsequent follow-up scans showed resolution of the EPN.

Conclusions: EPN is a severe, necrotizing infection of the kidneys associated with high mortality rates. As such, physicians need to have a high index of suspicion in patients who are at risk. There is increasing evidence to show that patients with early EPN can and should be treated with appropriate IV antibiotics and percutaneous drainage procedures. We hope that this will help to increase awareness of the possible treatment modalities of this potentially life-threatening disease.
\end{abstract}

Key Words: Emphysematous pyelonephritis, Percutaneous drainage, Necrotizing infection, Conservative

\section{INTRODUCTION}

Emphysematous pyelonephritis (EPN) was first described in 1898 by Kelly and MacCallum. It is a rare but severe necrotizing infection of the renal parenchyma. EPN has been shown to be associated with a high rate of mortality, ${ }^{[1]}$ primarily secondary to sepsis.

Traditionally, patients were treated with medical therapy along with early emergency nephrectomy and/or open surgical drainage. Reported mortality with this standard approach was high at $40 \%-50 \%{ }^{[2]}$ However, in recent years, there has been a shift in treatment practices with an increasing number of patients being treated with a less invasive approach of percutaneous drainage. Despite this, when faced with a patient, the clinical decision with respect to surgical versus non-surgical management and when to intervene with a nephrectomy, can be a difficult one to make. We present a case with extensive EPN on imaging scans but was successfully treated with appropriate intravenous antibiotics (IV) and percutaneous drainage.

\footnotetext{
*Correspondence: Jun Yang Chia; Email: yang_chia@nuhs.edu.sg; Address: National University Hospital, 5 Lower Kent Ridge Road, 119 074, Singapore.
}

Published by Sciedu Press 


\section{CASE REPORT}

Mr K was a 75-year-old Indian gentleman with a history of type II diabetes mellitus, ischaemic heart disease as well as a right renal calculus. He presented with severe right iliac fossa pain. His vital signs were stable on admission and he was afebrile. Examination was unremarkable except for right iliac fossa tenderness and a positive right renal punch. There was otherwise no abdominal rigidity or guarding. His white cell count was not markedly elevated at $10.39 \times 10^{9} / \mathrm{L}$. His creatinine level was elevated at $246 \mu \mathrm{mol} / \mathrm{L}$ on admission, giving an estimated glomerular filtration rate of $22 \mathrm{ml} / \mathrm{min}$ (by Modification Diet Renal Disease [MDRD] equation).

A computer tomography (CT) scan of the kidneys, ureter and bladder showed a significant amount of gas within the right renal parenchyma and pelvicalyceal system, tracking to the retroperitoneal region. There was also a right renal pelvis calculus measuring $13.18 \mathrm{~mm} \times 15.33 \mathrm{~mm}$ and a right mid ureter calculus measuring $9.18 \mathrm{~mm} \times 9.30 \mathrm{~mm}$ (see Figure 1, Figure 2).

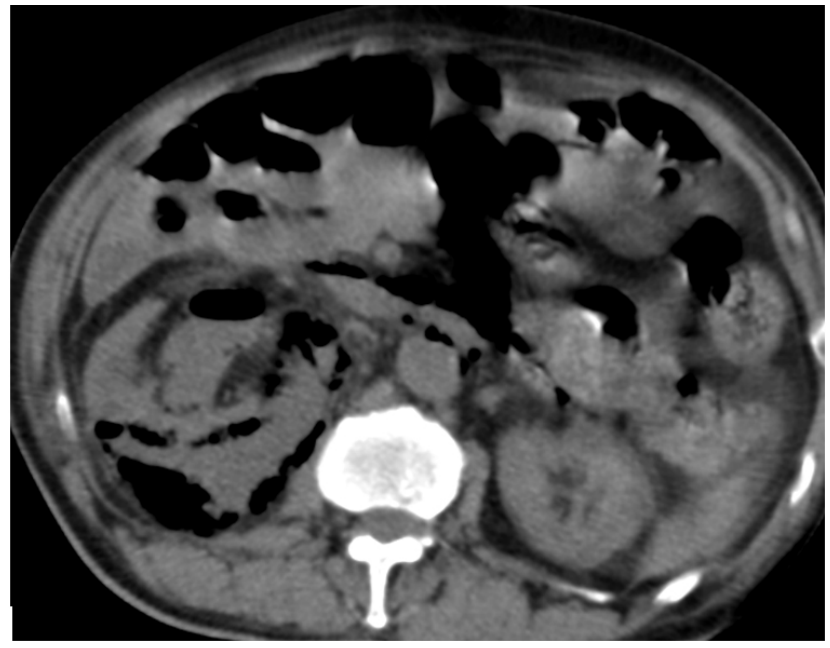

Figure 1. Free gas in right renal parenchyma

Urine cultures grew multi-resistant Escherichia Coli sensitive to Imipenam. Despite the extensive gas finding on his CT scan, in view of his clinical stability, it was elected to treat him with intravenous Ertapenam and to plan for an ultrasound guided percutaneous tube insertion to drain the intraparenchymal gas which can cause renal injury due to pressure effects. Other considerations against emergency nephrectomy included his comorbidities and likelihood of dialysis after a nephrectomy. Post procedure, repeat CT scan showed interval improvement in the EPN (see Figure 3). He remained clinically stable with an improvement in his pain score.

Mr K completed two weeks of intravenous Ertapenam and his creatinine levels stabilized at $187 \mu \mathrm{mol} / \mathrm{L}$ upon discharge. On follow up at one month in the outpatient clinic, a repeat CT scan showed further interval improvement with a decrease in the amount of gas in the right kidney and retroperitoneal region (see Figure 4), and his nephrostomy tube was removed then. Four months later, both the right renal and mid ureteric calculi were removed via standard endoscopy and laser lithotripsy under general anaesthesia.

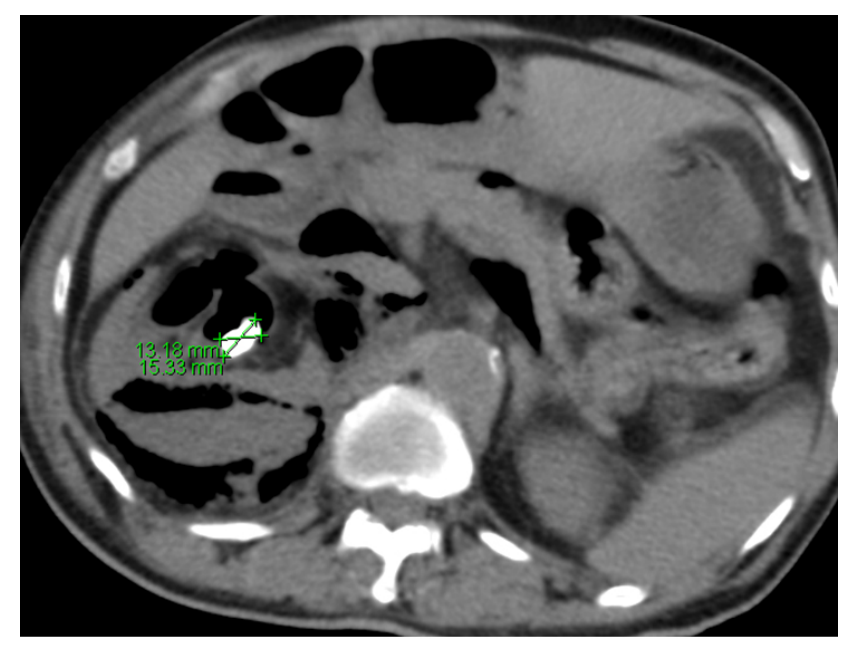

Figure 2. Calculus in right renal pelvis measuring $13.18 \mathrm{~mm} \times 15.33 \mathrm{~mm}$

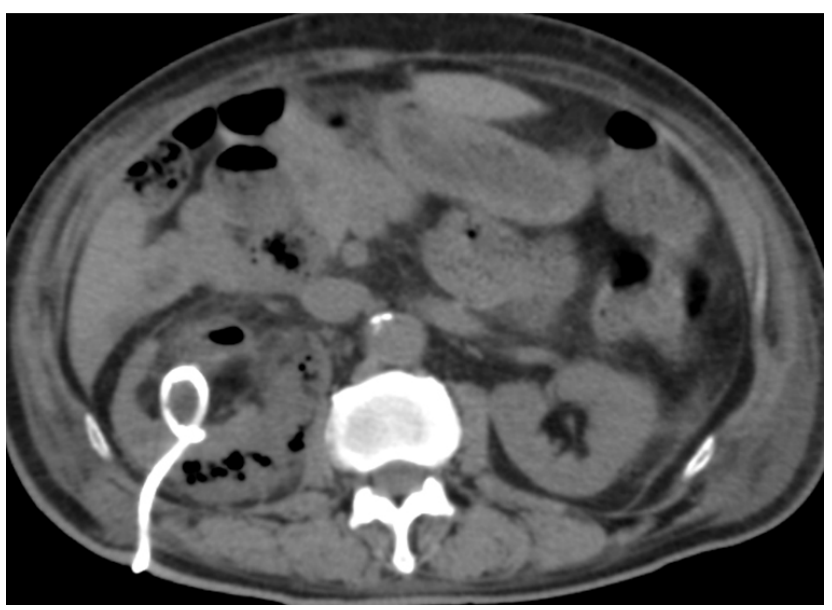

Figure 3. Interval improvement after percutaneous tube insertion

\section{Discussion}

As many as $90 \%$ of cases of EP are in patients with diabetes mellitus. Other risk factors include obstruction in the urinary tract, polycystic kidney disease, impaired vascular supply, presence of gas-forming organisms and patients on immunosuppression. ${ }^{[3]}$ 


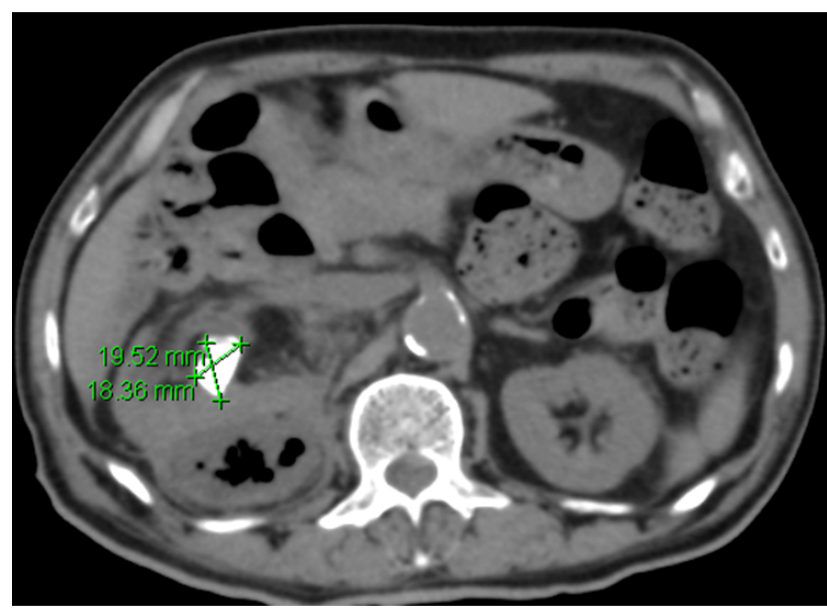

Figure 4. Further interval improvement on follow up at one month

Patients commonly present with fever associated with rigors, flank pain, nausea and vomiting, dysuria, hematuria and pyuria. They may rapidly progress to septic shock. The most common causative agents for EPN is Escherichia. Coli (50\%-70\%), followed by Klebsiella, Enterobacter, Proteus and Pseudomonas species. ${ }^{[2]}$ Infrequently, fungi like Candida, Cryptococcus and Aspergillus can cause EPN, and polymicrobial infection is seen in $14 \%-19 \%$ of cases. ${ }^{[2,4]}$

Diagnosis of EPN is based on radiological scans. Abdominal ultrasonography and plain radiograph are accurate in only $69 \%$ and $65 \%$ of cases respectively. ${ }^{[5]}$ Therefore confirmation of diagnosis should be based on findings from a CT scan which is also useful for substantiating the disease. ${ }^{[6]}$ Gas found on CT scan is characteristic and is produced under anaerobic conditions by gas-forming organisms mentioned above. In particular, the degree of extension of gas was reported to be an independent predictor of EPN prognosis. ${ }^{[6]}$

Hence, percutaneous drainage and antibiotics can be a modality of treatment for patients with limited EPN while early judicious nephrectomy should be reserved for patients with extensive EPN. In the above case, although Mr K presented with an aggressive $\mathrm{CT}$ picture of extensive retroperitoneal gas, he responded well to percutaneous drainage and antibiotics. Despite the old dogma of emergency nephrectomy for patients with extensive EPN, the above case illustrates that the management of these patients should be based on the clinical stability and according to Hippocrates oath - Do no harm first. As described, the above patient was also at an increased risk of morbidity and mortality under general anaethesia in view of his pre-existing comorbidites and the emergency septic setting. In planning for surgery, due consideration should also be given to patients with chronic kidney disease as the resultant renal reserve after nephrectomy might be inadequate to prevent dialysis dependence.

\section{Conclusion}

In conclusion, EPN is a potentially life-threatening condition but consideration of the treatment options should be based not only on the extent of gas on CT scan but also the patient's clinical picture.

\section{REFERENCES}

[1] Lin YC, Lin YC, Lin HD, et al. Risk factors of Renal Failure and severe complications in patients with emphysematous pyelonephritis - a single-center 15-year experience. Am J Med Sci. 2012; 343(3): 186-91. PMid: 21804369. http://dx.doi .org/10 .1097/MAJ . 0 b013e318225b891

[2] Michaeli J, Mogle S, Perlberg S, et al. Emphysematous pyelonephritis. J Urol. 1984; 131: 203-208. PMid: 6366247.

[3] Ahmad M, Dakshinamurty KV. Emphysematous renal tract disease due to Aspergillus fumigatus. J Assoc Phys Ind. 2004; 52: 495-497.

[4] Somani BK, Nabi G, Thorpe P, et al. Is percutaneous drainage the new gold standard in the management of emphysematous pyelonephritis? Evidence from a systemic review. J Urol. 2008; 179: 1844-9. PMid: 18353396. http://dx.doi.org/10.1016/j.juro. 2008 .01 .019

[5] Dutta P, Bhansali A, Singh SK, et al. Presentation and outcome of emphysematous renal tract disease in patients with diabetes mellitus. UrolInt. 2007; 78(1): 12-22. http://dx.doi.org/10.1159/000 096929

[6] Huang JJ, Chin-Chung, Tseng CC. Emphysematous pyelonephritis clinical classification, management, prognosis and pathogenesis. Arch Intern Med. 2000; 160: 797-805. PMid: 10737279. http://dx.doi.org/10.1001/archinte.160.6.797 